\title{
Engineering Oriented Heme Protein Maquette Monolayers through Surface Residue Charge Distribution Patterns
}

\author{
Xiaoxi Chen, Christopher C. Moser, Denis L. Pilloud, Brian R. Gibney, and P. Leslie Dutton* \\ Johnson Research Foundation, Department of Biochemistry and Molecular Biophysics, \\ University of Pennsylvania, Philadelphia, Pennsylvania 19104
}

Received: June 17, 1999; In Final Form: August 23, 1999

\begin{abstract}
We have designed and synthesized four- $\alpha$-helix-bundle proteins that accommodate heme groups to act as molecular "maquettes" of more complex natural electron-transfer proteins. These bundles can be oriented at an air-water interface and transferred onto solid surfaces to facilitate the exploration of the factors that govern biological electron transfer. We find that the orientation of these maquettes on an air-water interface can be controlled by choosing the distribution of charged amino acids along the sides of the helices exposed to water. The four $\alpha$-helices were assembled either as two subunits, where each subunit consists of two $\alpha$-helices linked by a terminal cysteine disulfide bond, or as a single, four-helix covalent unit consisting of two helix -loop-helix molecules linked by a terminal cysteine. In either case, when each $\alpha$-helix contains both positively charged lysines and negatively charged glutamates, addition of the heme binding bundles to an air-water interface causes them to open up and lie on the surface with $\alpha$-helical axes oriented parallel to the interface. In contrast, when the positive and negative charges are segregated on different helices (two negative, two positive) of the single covalent four- $\alpha$-helix-bundle unit, the bundle preserved its integrity on transfer to the air-water interface. Moreover, the presence of heme dictates the orientation of the $\alpha$-helical axes of the bundle with respect to the surface plane. The $\alpha$-helices adopt a parallel orientation in the absence of heme and a perpendicular orientation in the presence of heme. Circular dichroism (CD) and ultravioletvisible (UV-vis) spectroscopy supported by linear dichroism demonstrate that these molecular orientations are preserved in Langmuir-Blodgett monolayer films on solid substrate surfaces.
\end{abstract}

\section{Introduction}

A novel approach to the study of complex natural oxidoreductase enzymes is to design and synthesize proteins with the minimal requirements for the assembly and incorporation of active redox cofactors. These de novo designed ${ }^{1-3}$ minimalist redox proteins act as molecular "maquettes" to uncover the engineering behind the assembly of natural oxidoreductases that promote directed, long-range electron transfer. ${ }^{4,5} \mathrm{We}$ choose sequences strongly favoring $\alpha$-helical folding to present hydrophobic residues along one face of each helix and charged groups on the opposite face. Hydrophobic forces drive association of the helices into a four- $\alpha$-helix bundle in solution, while histidines in the bundle core ligate added hemes. These heme protein maquettes have proven to possess the characteristic electrochemical and optical properties typical of natural $b$-type cytochromes. ${ }^{6-10}$

Maquettes can be engineered to organize on surfaces by exploiting the great freedom of de novo design and chemical synthesis to modify exposed residues and control the interactions between $\alpha$-helices. Organizing maquettes on surfaces ${ }^{11,12}$ potentially not only facilitates electrochemical studies, ${ }^{13-15}$ which explore the factors that govern biological electron tunneling, but also forms the foundation for translating these heme protein maquettes into novel functional biomaterials. ${ }^{16-18}$ Furthermore, study of the surface assembly of these heme protein maquettes provides information on the nature of the various interactions between proteins and membranes or artificial surfaces. ${ }^{19}$

* To whom correspondence should be addressed. Fax: (215) 573-2235 E-mail: dutton@mail.med.upenn.edu.

10.1021/jp992000v CCC: \$18.00 두 1999 American Chemical Society
In a previous study, ${ }^{12}$ we explored the Langmuir-Blodgett (LB) film properties of our prototype four- $\alpha$-helix-bundle hemebinding protein maquettes. We showed that this homodimeric prototype heme protein $(\alpha \text {-ss- } \alpha)_{2}$ shown in Figure 1A dissociates on the air-water interface into its monomeric subunits, $\alpha$-ss$\alpha$, and orients its $\alpha$-helical axes parallel to the surface, presumably with the hydrophobic interior of the original four$\alpha$-helix bundle exposed to the air. We also showed that the addition of hydrophobic tails, such as palmitic acid, to the $\mathrm{N}$-termini of the $(\alpha-\mathrm{ss}-\alpha)_{2}$ heme protein induces reorientation at high surface pressure such that the $\alpha$-helices adopt an angle perpendicular to the air-water interface. Encouraged by the observation that the polarity distribution on the exterior of the bundles can influence the structures on the air-water interface, we have focused on the effect of altering exterior charge distributions; three related synthetic heme protein maquettes are compared.

1. $(\alpha-s s-\alpha)_{2}$. The $(\alpha-s s-\alpha)_{2}$ heme protein has been described in the previous study. ${ }^{12}$ It is a highly water-soluble four- $\alpha$-helixbundle heme protein that is based on the prototype described by Robertson et al., ${ }^{6}$ conservatively modified to improve packing of the bundle interior ${ }^{20}$ (see Materials and Methods). In the bundle, a pair of identical $\alpha$-helices are linked by a disulfide bond at their cysteinyl N-termini to make an $\alpha$-ss- $\alpha$ subunit. A pair of $\alpha$-ss- $\alpha$ subunits spontaneously assemble in aqueous solution to form a noncovalent four- $\alpha$-helix bundle $(\alpha \text {-ss- } \alpha)_{2}$. On one side of each $\alpha$-helix there are seven glutamates, six lysines, and one arginine forming alternate positive and negative patches, which form a candy cane striped pattern on the exterior 


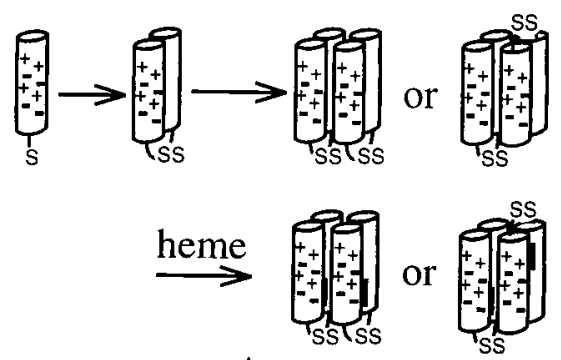

A

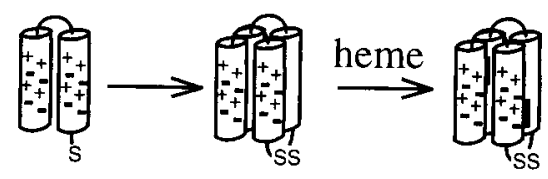

$\mathrm{B}$

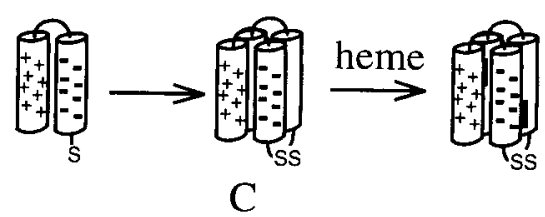

Figure 1. Design and construction of heme protein maquettes. Part A shows the construction of heme $2-(\alpha-s s-\alpha)_{2}$; the solid-phase peptide synthesis produced the 31 -amino-acid peptide $\alpha$, which is oxidized to form a dihelix unit $\alpha$-ss- $\alpha$. Two $\alpha$-ss- $\alpha$ units self-assemble into a fourhelix bundle $(\alpha-s s-\alpha)_{2}$. The four-helix bundle coordinates hemes by bis-histidyl ligation to form the heme protein maquette heme ${ }_{2}-(\alpha$-ss$\alpha)_{2}$. Part B shows the construction of heme $e_{2}(\alpha-\mid-\alpha-s-)_{2}$; the solid-phase peptide synthesis produced the 63 -amino-acid peptide $\alpha-\mid-\alpha$, which is oxidized to form the four-helix bundle $(\alpha-\mid-\alpha-s-)_{2}$. The four-helix bundle coordinates hemes by bis-histidyl ligation to form the heme protein maquette heme $2_{2}-(\alpha-l-\alpha-s-)_{2}$. Part $C$ shows the construction of heme ${ }_{2-}$ $\left(\alpha^{+}-1-\alpha^{-}-s-\right)_{2}$; the solid-phase peptide synthesis produced the 63-aminoacid peptide $\alpha^{+}-\left.\right|_{-} \alpha^{-}$(with lysines and glutamates segregated on different helices), which is oxidized to form the four-helix bundle $\left(\alpha^{+}-\right.$ $\left.\mathrm{I}-\alpha^{-}-\mathrm{s}-\right)_{2}$. The four-helix bundle coordinates hemes by bis-histidyl ligation to form the heme protein maquette, heme $2_{2}\left(\alpha^{+}-\mid-\alpha^{-}-\mathrm{s}-\right)_{2}$.

of the assembled four- $\alpha$-helix bundle. Figure 1A shows that the four- $\alpha$-helix bundle can adopt a syn or anti topology in which the disulfide bonds are at the same or opposite ends of the bundle, depending on the sequence and cofactor binding. ${ }^{21-23}$ The structure of this four- $\alpha$-helix bundle has been studied by NMR spectra. ${ }^{24,25}$

2. $(\alpha-\mid-\alpha-s-)_{2}$. The amino acid sequence of each $\alpha$-helix of the $(\alpha-\mid-\alpha-s-)_{2}$ heme protein is identical to that of the $(\alpha-s s-\alpha)_{2}$ heme protein. However, as shown in Figure 1B, two repeating $\alpha$-helix amino acid sequences with six glycines between are synthesized as a single sequence. A pair of single sequence $\alpha-\mid-\alpha$ are linked by a disulfide bond at their cysteinyl $\mathrm{N}$-termini to form a covalent four- $\alpha$-helix bundle $(\alpha-\mid-\alpha \text {-s- })_{2}$. The external charges of $(\alpha-\mid-\alpha-s-)_{2}$ and $(\alpha-\text { ss- } \alpha)_{2}$ are similar.

3. $\left(\alpha^{+}-\mid-\alpha^{-}-\mathrm{s}-\right)_{2}$. The $\left(\alpha^{+}-\mid-\alpha^{-}-\mathrm{s}-\right)_{2}$ heme protein is a variant of the $(\alpha-\mid-\alpha-s-)_{2}$ heme protein with a major change in the distribution of the exterior charged residues compared with $(\alpha-$ $\mid-\alpha$-s- $)_{2}$ and $(\alpha-s s-\alpha)_{2}$. In $\left(\alpha^{+}-\mid-\alpha^{-}-s-\right)_{2}, 14$ glutamates are placed in one helix and 14 lysines in the other helix to form a highly asymmetrically charged $\alpha^{+}-\mid-\alpha^{-}$, as shown in Figure 1C. Then, as with the $(\alpha-\mid-\alpha-s-)_{2}$, a pair of $\alpha^{+}-\left.\right|_{-} ^{-} \alpha^{-}$are linked by a disulfide bond at their cysteinyl $\mathrm{N}$-termini to form a four- $\alpha$-helix bundle $\left(\alpha^{+}-\mid-\alpha^{-}-\mathrm{s}-\right)_{2}$.

This report explores the properties of the three heme protein maquettes in a Langmuir-Blodgett film balance and after deposition on planar substrate surfaces. We show that the $(\alpha$ ss- $\alpha)_{2}$ and $(\alpha-\mid-\alpha-s-)_{2}$ heme proteins display similar properties on the air-water interface while the $\left(\alpha^{+}-\mid-\alpha^{-}-\mathrm{s}-\right)_{2}$ heme protein is dramatically different. It is clear that the segregated charge distribution of $\left(\alpha^{+}-\mid-\alpha^{-}-s-\right)_{2}$ increases the four- $\alpha$-helix-bundle integrity through strong electrostatic interactions between the helices and provides a basic maquette design adapted for both solutions and interfaces.

\section{Materials and Methods}

Peptide Sequences. 1. $(\alpha-s s-\alpha)_{2}$. The amino acid sequence of each $\alpha$-helix in the $(\alpha-s s-\alpha)_{2}$ heme protein is

\section{Ac-CGGG·EIWKLH·EEFLKKF•EELLKLF•}

EERLKKL-CONH

Histidine at position 10 is for heme binding. A pair of identical $\alpha$-helices are linked by the disulfide bond at their cysteinyl $N$-termini to make an $\alpha$-ss- $\alpha$ subunit. Two $\alpha$-ss- $\alpha$ subunits spontaneously assemble in aqueous solution to form a four- $\alpha$ helix bundle $(\alpha \text {-ss- } \alpha)_{2}$. This peptide differs from the prototype peptide ${ }^{6}$ H10A24 in the interior residues L6I, L13F, and A24F and, as shown by NMR, has a singular structure. ${ }^{25}$

2. $(\alpha-\mid-\alpha-s-)_{2}$. The amino acid sequence of the $\alpha-\mid-\alpha$ in the $(\alpha-\mid-\alpha-s-)_{2}$ heme protein is

\section{Ac-CGG・EIWKLH·EEFLKKF•EELLKLF•EERLKKL• $\mathrm{GGG} \cdot \mathrm{GGG} \cdot \mathrm{EIWKLH} \cdot \mathrm{EEFLKKF} \cdot \mathrm{EELLKLF} \cdot$ EERLKKL-CONH}

A pair of single sequence $\alpha-\mid-\alpha$ are linked by a disulfide bond at their cysteinyl $\mathrm{N}$-termini to form a four- $\alpha$-helix bundle $(\alpha$ $1-\alpha-s-)_{2}$.

3. $\left(\alpha^{+}-\mid-\alpha^{-}-s-\right)_{2}$. The amino acid sequence of the $\alpha^{+}-\left.\right|_{-} ^{-} \alpha^{-}$in the $\left(\alpha^{+}-\mid-\alpha^{-}-s-\right)_{2}$ heme protein is

\section{Ac-CGG·EIWELH·EEFLEEF•EELLELF•EEELEEL• GGG・GGG・KIWKLH•KKFLKKF•KKLLKLF• KKKLKKL-CONH}

A pair of $\alpha^{+}-\mid-\alpha^{-}$are linked by a disulfide bond at their cysteinyl $\mathrm{N}$-termini to form a four- $\alpha$-helix bundle $\left(\alpha^{+}-\mathrm{I}-\alpha^{-}-\mathrm{s}-\right)_{2}$.

Chemicals and Solvents. Hemin, trifluoroacetic acid (TFA), dimethyl sulfoxide (DMSO), and octadecyltrichlorosilane were purchased from Aldrich Chemical Co. (Milwaukee, WI). Ethanedithiol and 1-hydroxybenzotriazol were purchased from Fluka (Ronkonkoma, NY). FMOC-protected amino acid perfluorophenyl esters were purchased from PerSeptive Biosystems (Framingham, MA) with the exception of FMOC-L-Arg(Pmc)OPfp, which was obtained from Bachem (King of Prussia, PA). NovaSyn PR-500 resin was purchased from CalbiochemNovabiochem (La Jolla, CA). All other chemicals and solvents were reagent grade. Water was purified using a Milli-Q water system from Millipore Corp. (Bedford, MA).

Peptide Synthesis. The peptides were synthesized on a continuous-flow Milligen 9050 solid-phase synthesizer using standard FMOC $-{ }^{t} \mathrm{Bu}$ protection strategy with NovaSyn PR500 resin at $0.2 \mathrm{mmol}$ scale. The side chain protecting groups used are as follows: Lys(tBoc), Glu(OtBu), Cys(Trt), and $\operatorname{Arg}(\mathrm{Pmc})$. The peptides were cleaved from the resin and simultaneously deprotected using 90:8:2 trifluoroacetic acid/ ethanedithiol/water for $2 \mathrm{~h}$. Crude peptides were precipitated and triturated with cold ether, dissolved in water $(0.1 \% \mathrm{TFA})$, lyophilized, and purified to homogeneity by reversed-phase $\mathrm{C} 18$ HPLC using aqueous acetonitrile gradients containing $0.1 \%$ (v/ v) TFA. The resulting peptide identities were confirmed with laser desorption mass spectrometry. 
Heme Protein Maquette Preparation. 1. Heme ${ }_{2}(\alpha-s s-\alpha)_{2}$. The 31-amino-acid peptides were dissolved in $100 \mathrm{mM}$ ammonium hydrogen carbonate buffer $(\mathrm{pH} 9.2)$ in air to allow the cysteines to oxidize to form the 62-amino-acid peptide $\alpha$-ss- $\alpha$. After $4 \mathrm{~h}$ the solution was frozen and lyophilized. When needed, the $\alpha$-ss- $\alpha$ peptides were dissolved in phosphate buffer $(50 \mathrm{mM}$ phosphate, $100 \mathrm{mM} \mathrm{NaCl}, \mathrm{pH} 8.0)$ to yield a final concentration of $0.02 \mathrm{mM}(\alpha-s s-\alpha)_{2}$. Heme was incorporated into $(\alpha-s s-\alpha)_{2}$ to form heme $2_{2}(\alpha-s s-\alpha)_{2}$ from a stock solution of $4 \mathrm{mM}$ of $\mathrm{Fe}(\mathrm{III})$ protoporphyrin IX (heme) in DMSO by successive additions of 0.1 heme per binding site until 1 heme per bishistidine site was reached. During each addition, the solution was well stirred and then allowed to equilibrate for $5 \mathrm{~min}$. The final concentration of DMSO in the aqueous solution was always lower than 1:100 (v/v). The heme incorporation was monitored by the increase of the Soret band maximum between 411 and $412 \mathrm{~nm}\left(\epsilon=120000 \mathrm{M}^{-1} \mathrm{~cm}^{-1}\right)$.

2. Heme $_{2}-(\alpha-\mid-\alpha-s-)_{2}$. The 63-amino-acid $\alpha-I-\alpha-s$ peptides were dissolved in $100 \mathrm{mM}$ ammonium hydrogen carbonate buffer ( $\mathrm{pH}$ 9.2) in air to allow the cysteines to oxidize to form the 126 -amino-acid peptide $(\alpha-\mid-\alpha-s-)_{2}$. After $4 \mathrm{~h}$ the solution was frozen and lyophilized. When needed, the $(\alpha-\mid-\alpha-s-)_{2}$ peptides were dissolved in phosphate buffer $(50 \mathrm{mM}$ phosphate, $100 \mathrm{mM} \mathrm{NaCl}, \mathrm{pH} 8.0)$ to yield a final concentration of 0.02 $\mathrm{mM}(\alpha-\mathrm{I}-\alpha-\mathrm{s}-)_{2}$. Heme was incorporated into $(\alpha-\mathrm{l}-\alpha-\mathrm{s}-)_{2}$ to form heme $_{2}-(\alpha-\mid-\alpha-s-)_{2}$ with the same procedure described above.

3. Heme ${ }_{2}-\left(\alpha^{+}-\mid-\alpha^{-}-s-\right)_{2}$. The 63-amino-acid $\alpha^{+}-\mid-\alpha^{-}$-s peptides were dissolved in high ionic strength phosphate buffer (50 $\mathrm{mM}$ phosphate, $1.5 \mathrm{M} \mathrm{NaCl}$ ) at $\mathrm{pH} 4.4$ to yield a final concentration of $0.04 \mathrm{mM} \alpha^{+}-\mid-\alpha^{-}-\mathrm{s}$, and then the $\mathrm{pH}$ was adjusted to 8.0 by adding $\mathrm{NaOH}$. The peptide solution was then exposed to air to allow the cysteines to oxidize to form disulfide bonds and $\left(\alpha^{+}-\mid-\alpha^{-}-s_{-}\right)_{2}$. Heme was incorporated into $\left(\alpha^{+}-\mid-\right.$ $\left.\alpha^{-}-\mathrm{s}-\right)_{2}$ to form heme ${ }_{2}\left(\alpha^{+}-\mid-\alpha^{-}-\mathrm{s}-\right)_{2}$ following the same procedure described above.

Solution Molecular Weight Determination. Size exclusion chromatography was performed on a Beckman System Gold HPLC system with a diode array detector using a Supelco Sigmachrom GFC-100 column $(300 \mathrm{~mm} \times 57.5 \mathrm{~mm})$ eluted with aqueous buffer. The column was standardized using aprotinin $(6.5 \mathrm{kDa})$, ribonuclease $\mathrm{A}(13.7 \mathrm{kDa})$, chymotrypsinogen $(25.0 \mathrm{kDa})$, ovalbumin $(43.0 \mathrm{kDa})$, and bovine serum albumin $(67.0 \mathrm{kDa})$.

Langmuir Film Preparation and Isotherm Measurement. A Langmuir-Blodgett film balance (Lauda Filmbalance FW2; Sybron/Brinkmann, Westbury, NY) was used to make the films according to a general procedure described elsewhere. ${ }^{26,27}$ The aqueous subphase contained $1 \mathrm{mM}$ phosphate at $\mathrm{pH}$ 8.0. All films were made under an argon atmosphere at a subphase temperature of $22{ }^{\circ} \mathrm{C}$. Proteins were spread at the air-water interface using the "glass-rod" method. ${ }^{28}$ This involved placing a $0.6 \mathrm{~mm}$ diameter glass rod at a small angle $\left(<30^{\circ}\right)$ relative to the trough plane, just touching the water surface, to which 2 $\mathrm{mL}$ aliquots of material were added every $10-15 \mathrm{~s}$. During this procedure the trough surface area was held constant. Surface pressure-area isotherms were measured using a barrier speed of $10 \mathrm{~cm}^{2} / \mathrm{min}$.

Substrate Preparation. Quartz slides, $1 \mathrm{~mm}$ in thickness (Esco Products, Oak Ridge, NJ), were sonicated first in detergent solution and then in water. This was followed by immersion in a freshly prepared solution of 4:1 (v/v) 95-98\% sulfuric acid/ $30 \%$ hydrogen peroxide (CAUTION: This solution is highly oxidizing and very acidic and should be handled with extreme care) at room temperature for about $1 \mathrm{~h}$. The slides were then thoroughly rinsed with water and finally stripped of visible surface water with a stream of argon. The cleaned slides were then rendered hydrophobic by silanation with octadecyltrichlorosilane. This was achieved by sonicating the slides for 20 min in a $0.1 \%$ octadecyltrichlorosilane in a solution of $80 \%$ hexadecane ( $99 \%$ pure), $12 \%$ carbon tetrachloride, and $8 \%$ chloroform. The slides were washed three times in chloroform for at least $5 \mathrm{~min}$ for each wash. The quality of the hydrophobic coating was checked by placing a $20 \mathrm{~mL}$ droplet of water on the slide. The coating was considered satisfactory if the droplet started moving freely and continuously on the slide when the tilt angle of the glass exceeded $\sim 40^{\circ}$.

Langmuir-Blodgett Monolayer Film Preparation. Protein maquettes were spread as discussed above, compressed, and held at a constant surface pressure of $\sim 20 \mathrm{mN} / \mathrm{m}$. A hydrophobically modified quartz slide was passed vertically into the subphase at $3 \mathrm{~mm} / \mathrm{min}$. The submerged slide was then released into a vial resting at the bottom of the trough. After LB deposition, the slide was transferred from the vial to a quartz cuvette for spectroscopic characterization. The entire process was done in buffer so that the slide was never exposed to air.

Circular Dichroism Spectropolarimetry. Circular dichroism spectra were measured in an Aviv 62DS circular dichroism spectropolarimeter. Rectangular quartz cuvettes of $0.2 \mathrm{~cm}$ path length were used for measuring solution spectra. For film spectra, four quartz slides coated with LB film were positioned in a quartz cuvette of $1.0 \mathrm{~cm}$ path length using two Teflon blocks (each has four parallel slits and fits tightly on the bottom and top of the cuvette) holding the slides parallel to each other. The slides were immersed in buffer and oriented perpendicularly to the incident measuring beam. A baseline reference was recorded by using four blank, cleaned quartz slides positioned in the same way.

Ultraviolet-Visible Absorption Spectroscopy and Linear Dichroism. UV-vis absorption spectra were recorded with a Perkin-Elmer Lambda 2 UV-vis spectrophotometer. Rectangular quartz cuvettes of 0.2 and $1.0 \mathrm{~cm}$ path lengths were used for measuring solution spectra. For film spectra, quartz slides coated with LB film were positioned in a quartz cuvette of 1.0 $\mathrm{cm}$ path length using a Teflon block (which fits tightly on the bottom of the cuvette and has one slit at $0^{\circ}, 30^{\circ}, 45^{\circ}$, or $60^{\circ}$ angle relative to the edges of the block) holding the slide at a selected angle with respect to the incident measuring beam. The slides were always immersed in buffer. For linear dichroism measurements, a UV dichroic polarizer from Oriel Corporation (Stratford, CT) sensitive from 230 to $770 \mathrm{~nm}$ was placed between the incident beam and the coated slide. In all cases a baseline reference was recorded by using a blank, cleaned quartz slide.

\section{Results}

1. Solution Characterization of $\mathrm{Heme}_{2}-\left(\alpha^{+}-\mid-\alpha^{-}-\mathrm{s}-\right)_{2}$ Maquette. The $\left(\alpha^{+}-\mid-\alpha^{-}-s^{-}\right)_{2}$ protein maquette was found to have very different solubility properties from the $(\alpha \text {-ss- } \alpha)_{2}$ and $(\alpha-\mid-\alpha-s-)_{2}$ protein maquettes, evidently resulting from its highly asymmetric charge distribution. At ionic strengths up to $1 \mathrm{M}$, $\left(\alpha^{+}-\mathrm{I}^{-} \alpha^{-}-\mathrm{s}-\right)_{2}$ solutions are highly scattering in the $\mathrm{pH}$ range from 4.5 ( $\mathrm{p} K_{\mathrm{a}}$ of glutamate) to 10.2 ( $\mathrm{p} K_{\mathrm{a}}$ of lysine), as shown in parts $\mathrm{A}$ and $\mathrm{B}$ of Figure 2. However, solubility within this range increases with increasing ionic strength (parts $C$ and D of Figure 2). Thus, at an ionic strength of $1.5 \mathrm{M}$, the protein can be readily solvated even at neutral $\mathrm{pH}$, without causing precipitation. This is done by exposing the protein to $\mathrm{pH} 4.4$ 


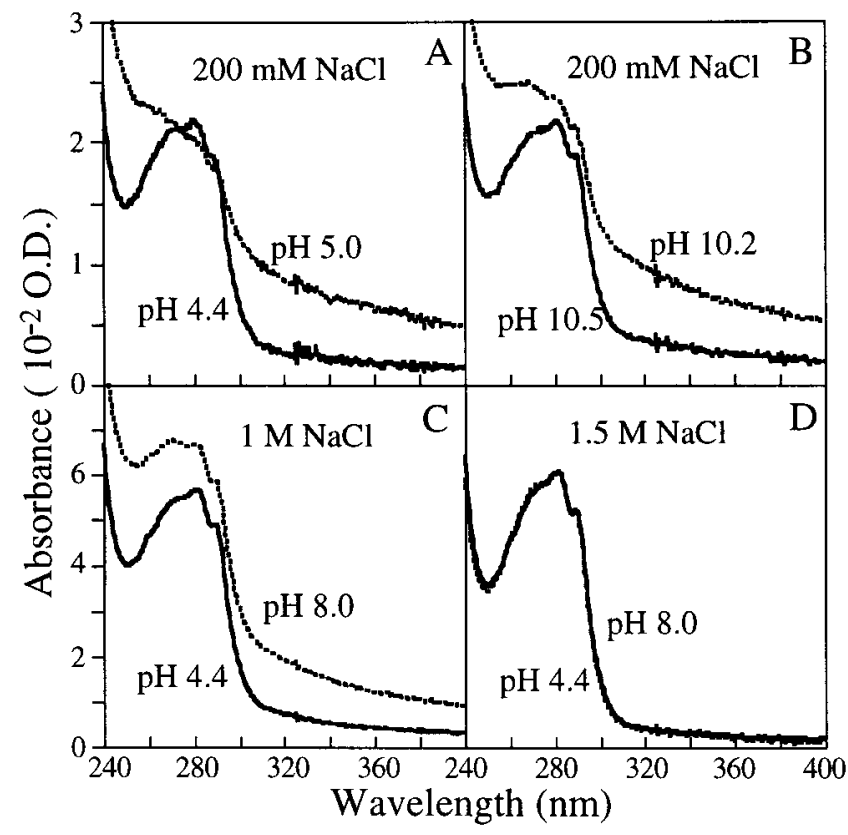

Figure 2. Solubility of $\left(\alpha^{+}-\mid-\alpha^{-}-s^{-}\right)_{2}$ at different $\mathrm{pH}$ and ionic strength, demonstrated by UV spectra of $\mu \mathrm{M}$ peptide solution. A clean tryptophan spectrum with a flat baseline near $400 \mathrm{~nm}$ indicates good solubility, while a broadened tryptophan spectrum with a slanted baseline indicates aggregation in the solution (also visible by looking through the solution). Part A shows that $\left(\alpha^{+}-\left.\right|_{-} \alpha^{-}-\mathrm{s}-\right)_{2}$ was soluble in $200 \mathrm{mM} \mathrm{NaCl}$ buffer at $\mathrm{pH}=4.4$ (where many glutamates are neutral) but aggregated when the $\mathrm{pH}$ was adjusted to 5.0. Part B shows that $\left(\alpha^{+}-\mathrm{I}-\alpha^{-}-\mathrm{s}-\right)_{2}$ was soluble in $200 \mathrm{mM} \mathrm{NaCl}$ buffer at $\mathrm{pH}=10.5$ (where many lysines are neutral) but aggregated when the $\mathrm{pH}$ was adjusted to 10.2. Part $\mathrm{C}$ shows that $\left(\alpha^{+}-\mid-\alpha^{-}-\mathrm{s}-\right)_{2}$ was soluble in $1 \mathrm{M} \mathrm{NaCl}$ buffer at $\mathrm{pH}=4.4$, and some aggregation was observed when the $\mathrm{pH}$ was adjusted to 8.0. Part D shows that $\left(\alpha^{+}-\mid-\alpha^{-}-\mathrm{s}-\right)_{2}$ was soluble in $1.5 \mathrm{M} \mathrm{NaCl}$ buffer at $\mathrm{pH}=$ 4.4 , and no aggregation was observed when the $\mathrm{pH}$ was adjusted to 8.0.

buffer with $1.5 \mathrm{M} \mathrm{NaCl}$ followed by raising the $\mathrm{pH}$ to 8.0 (Figure 2D). Heme can be incorporated into the maquette in the high ionic strength solution thus prepared. The apparent molecular weights at high ionic strength determined by gel permeation chromatography were 17.3 and $18.2 \mathrm{kDa}$ before and after incorporation of hemes, which are very similar to the $(\alpha-$ ss- $\alpha)_{2}$ and heme ${ }_{2}(\alpha-\text {-ss- } \alpha)_{2}$ maquettes, and consistent with a four- $\alpha$-helix-bundle aggregation state for both $\left(\alpha^{+}-\mid-\alpha^{-}-s^{-}\right)_{2}$ and heme $_{2}-\left(\alpha^{+}-\mid-\alpha^{-}-s_{-}\right)_{2}$. Figure $3 A$ shows that heme ${ }_{2}-\left(\alpha^{+}-\left.\right|_{-} \alpha^{-}-s-\right.$ )$_{2}$ has a CD spectrum typical of a highly $\alpha$-helical structure with minima at 208 and $222 \mathrm{~nm}$, and the helical content $(75 \%)$ is similar to those of heme $2-(\alpha-s s-\alpha)_{2}$ and heme $2-(\alpha-\mid-\alpha-s-)_{2}$ maquettes. Figure $3 B$ shows that heme $2^{-}\left(\alpha^{+}-\mid-\alpha^{-}-s^{-}\right)_{2}$ has a $\mathrm{UV}-$ vis spectrum typical of bis-histidine ligated ferric heme with a Soret maxima at $412 \mathrm{~nm}$ and $\alpha$-band maxima at 550 $\mathrm{nm}$.

2. Characterization of the Maquettes on Air-Water Interface. The pressure-area isotherms of $(\alpha-s s-\alpha)_{2},(\alpha-\mid-\alpha-$ $\mathrm{s}-)_{2}$, and $\left(\alpha^{+}-\mid-\alpha^{-}-\mathrm{s}-\right)_{2}$ without heme are compared in Figure $4 \mathrm{~A}$, and the pressure-area isotherms of the heme-containing heme $_{2}-(\alpha-s s-\alpha)_{2}$, heme ${ }_{2}-(\alpha-\mid-\alpha-s-)_{2}$, and heme ${ }_{2}\left(\alpha^{+}-\mid-\alpha^{-}-s-\right)_{2}$ are compared in Figure 4B. The isotherms of all three maquettes share the characteristics that when the films were compressed to approximately $30 \mathrm{mN} / \mathrm{m}$, a transition from gaseous state to liquid expanded state of the monolayer occurs; beyond the transition point, the surface pressure increases only to a small extent per unit decrease of surface area. The surface area per molecule at the transition point provides a measure of the molecular area for orientations found at low surface pressure.
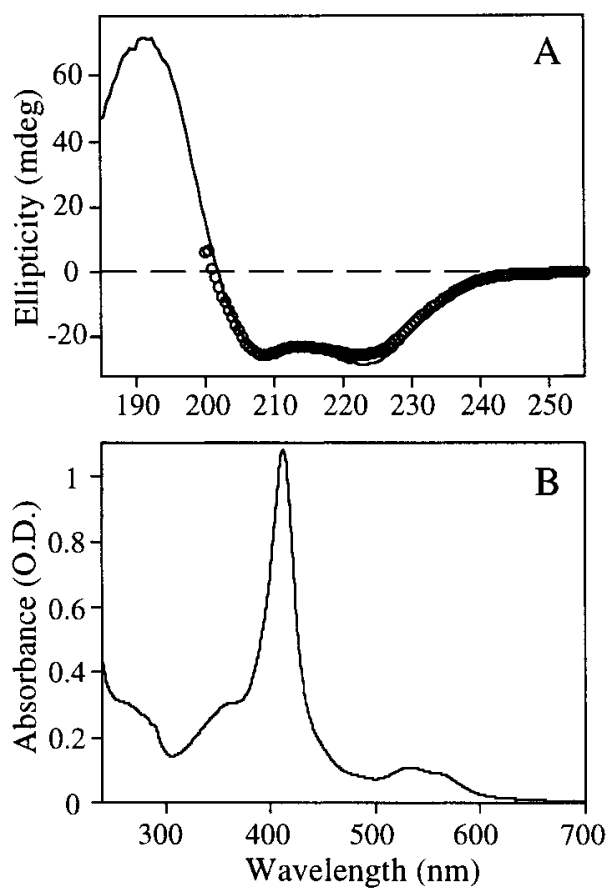

Figure 3. Characterization of heme $2^{-}\left(\alpha^{+}-\mid-\alpha^{-}-s^{-}\right)_{2}$ in $10 \mathrm{mM}$ phosphate, $1.5 \mathrm{M} \mathrm{NaCl}, \mathrm{pH} 8.0$ buffer. Part A shows a comparison of CD spectra of heme ${ }_{2}-\left(\alpha^{+}-\mid-\alpha^{-}-s-\right)_{2}$ (open circles) and heme ${ }_{2}-(\alpha-s s-\alpha)_{2}$ (solid line). For heme $2-\left(\alpha^{+}-\mid-\alpha^{-}-\mathrm{s}-\right)_{2}$, the CD spectropolarimeter was saturated at wavelengths below $200 \mathrm{~nm}$ because of the high absorption of UV light by the high concentration salt. Part B shows the UV-vis absorption spectrum of heme ${ }_{2}\left(\alpha^{+}-\mid-\alpha^{-}-s-\right)_{2}$.

In the low surface pressure gaseous state, a two-dimensional version of the ideal gas law applies: ${ }^{29}$

$$
\pi\left(A-A_{0}\right)=k T / n
$$

where $\pi$ is the surface pressure, $A$ the surface area per $\alpha$-helix, $A_{0}$ the limiting area per $\alpha$-helix, $k$ the Boltzmann constant, $T$ the temperature in kelvin, and $n$ the number of $\alpha$-helices per independent particle. In Figure $4 \mathrm{D}, k T / \pi$ values are plotted against $A$ for the low-pressure region (from 1 to $4 \mathrm{mN} / \mathrm{m}$ ) of each isotherm. The slopes of the fitted lines give the value of $n$, and the $x$-intercepts of the lines give the value of $A_{0}$. We compare this area with the dimensions of a single helix derived from an NMR structure, ${ }^{24}$ which has a helical diameter of $12-$ $13 \AA$, for a small cross section of $144-170 \AA^{2}$, and a helical length of $50 \AA$, for a lengthwise cross section of about $600 \AA^{2}$.

1. $(\alpha-s s-\alpha)_{2}$. Trace a in Figure 4A shows the isotherm of $(\alpha-$ ss $-\alpha)_{2}$, and Figure 4D reveals that its $n$ value is 2.09 , which indicates that two $\alpha$-helices are associated in the independent particles on the air-water interface. Figure 4D also reveals that the $A_{0}$ for the $(\alpha \text {-ss- } \alpha)_{2}$ film is $593 \AA^{2} / \alpha$-helix, which indicates that the axes of the $\alpha$-helices are parallel to the air-water interface. Thus, the four- $\alpha$-helix bundle appears to be dissociated into a pair of $\alpha$-ss- $\alpha$ subunits that are lying flat on the airwater interface, presumably with all polar sides in contact with the water surface and hydrophobic faces exposed to the air.

Trace $\mathrm{b}$ in Figure 4B shows the isotherm of heme ${ }_{2}(\alpha-s s-$ $\alpha)_{2}$, and Figure 4D reveals that its $n$ value is 2.05 and $A_{0}$ value is $602 \AA^{2} / \alpha$-helix, indicating that the maquette both with and without heme exhibits the same behavior on the air-water interface.

2. $(\alpha-\mid-\alpha-s-)_{2}$. Trace $\mathrm{c}$ in Figure $4 \mathrm{~A}$ shows the isotherm of $(\alpha-\mid-\alpha-s-)_{2}$, and Figure 4D reveals that its $n$ value is 4.06, which indicates as expected that the four $\alpha$-helices are associated in 


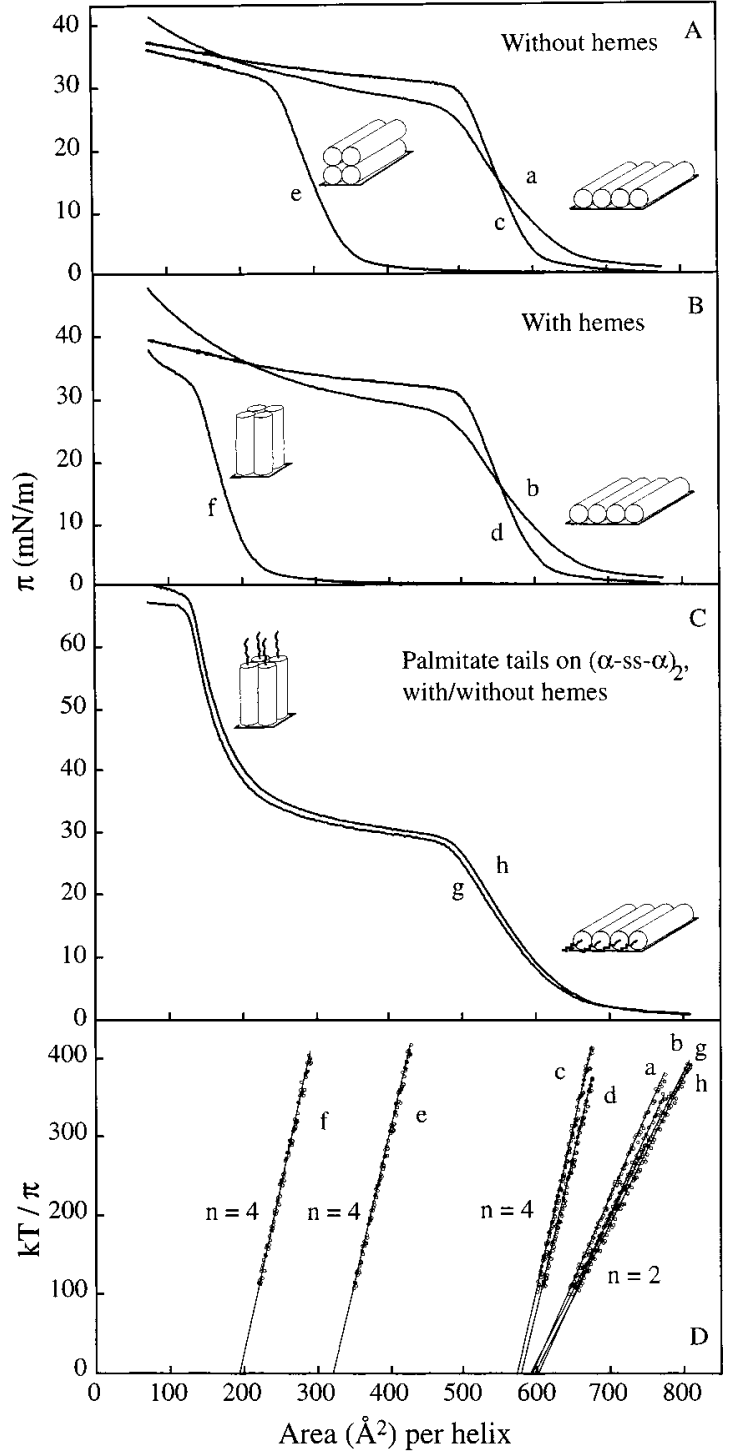

Figure 4. Orientation of maquettes on the air-water interface characterized by surface pressure-area isotherms. The subphase is 1 $\mathrm{mM}$ phosphate buffer (with no salt), $T=22{ }^{\circ} \mathrm{C}$. Part A shows the $\pi-A$ isotherms of the monolayer films derived from three maquettes without heme: $(\alpha-\text { ss- } \alpha)_{2}(\mathrm{a}),(\alpha-\mid-\alpha-s-)_{2}(\mathrm{c})$, and $\left(\alpha^{+}-\mathrm{l}-\alpha^{-}-\mathrm{s}-\right)_{2}(\mathrm{e})$. Part B shows the $\pi-A$ isotherms of the monolayer films derived from three maquettes with heme: heme $2_{2}(\alpha-s s-\alpha)_{2}(b), h^{2} e_{2}-(\alpha-I-\alpha-s-)_{2}(d)$, and heme $_{2}-\left(\alpha^{+}-\mid-\alpha^{-}-s-\right)_{2}$ (f). Part $C$ shows the $\pi-A$ isotherms of the monolayer films derived from $\left(\mathrm{palm}_{2}-\alpha-\mathrm{ss}-\alpha\right)_{2}(\mathrm{~g})$ and heme ${ }_{2}-\left(\mathrm{palm}_{2}-\right.$ $\alpha$-ss- $\alpha)_{2}$ (h) (characterized in ref 12). Part D shows plots of $R T / \pi$ versus $A$ for $\pi$ from 1 to $4 \mathrm{mN} / \mathrm{m}$. The experimental points (open circles) are fitted with linear equations $R T / \pi=n\left(A-A_{0}\right)$ (solid lines): (a) $(\alpha-$ ss- $\alpha)_{2}, n=2.09, A_{0}=593 \AA^{2} / \alpha$-helix; (b) heme ${ }_{2}(\alpha \text {-ss- } \alpha)_{2}, n=2.05$, $A_{0}=602 \AA^{2} / \alpha$-helix; (c) $(\alpha-l-\alpha-s-)_{2}, n=4.06, A_{0}=573 \AA^{2} / \alpha$-helix; (d) heme $2-(\alpha-\mathrm{l}-\alpha-\mathrm{s}-)_{2}, n=3.91, A_{0}=580 \AA^{2} / \alpha$-helix; (e) $\left(\alpha^{+}-\mathrm{l}-\alpha^{-}\right.$-s)$_{2}, n=3.89, A_{0}=322 \AA^{2} / \alpha$-helix; (f) heme ${ }_{2}\left(\alpha^{+}-\mid-\alpha^{-}-\mathrm{s}-\right)_{2}, n=4.19$, $A_{0}=194 \AA^{2} / \alpha$-helix; (g) $\left(\text { palm }_{2}-\alpha \text {-ss- } \alpha\right)_{2}, n=1.81, A_{0}=590 \AA^{2} / \alpha-$ helix; (h) heme 2 -(palm $2-\alpha-s s-\alpha)_{2}, n=1.83, A_{0}=598 \AA^{2} / \alpha$-helix. Orientations of the maquettes on the air-water interface that are consistent with the observed $n$ and $A_{0}$ values are illustrated near the isotherms in Parts A-C.

the independent particles on the air-water interface. Figure 4D also reveals that the $A_{0}$ for $(\alpha-\mid-\alpha-s-)_{2}$ is $573 \AA^{2} / \alpha$-helix, which also approximately coincides with the area of each $\alpha$-helix if they are parallel to the air-water interface. Thus, although the two $\alpha-\mid-\alpha$-s units are still linked together by the disulfide bond, they do not form a fully associated bundle but rather lie flat side-by-side or end-to-end on the air-water interface with polar faces down and hydrophobic faces to the air as discussed for $(\alpha \text {-ss- } \alpha)_{2}$ maquettes.

Trace $d$ in Figure 4B shows the isotherm of heme $2-(\alpha-\mid-\alpha-$ $\mathrm{s}-)_{2}$, and Figure 4D reveals that its $n$ value is 3.91 and $A_{0}$ value is $580 \AA^{2} / \alpha$-helix, indicating that the maquette both with and without heme exhibits the same behavior on the air-water interface.

3. $\left(\alpha^{+}-\mid-\alpha^{-}-s^{-}\right)_{2}$. Trace e in Figure 4A shows the isotherm of $\left(\alpha^{+}-\mid-\alpha^{-}-\mathrm{s}-\right)_{2}$, and Figure 4D reveals that its $n$ value is 3.89, which indicates as expected that the four $\alpha$-helices are associated in the independent particles on the air-water interface. However, Figure $4 \mathrm{D}$ also reveals that the $A_{0}$ for $\left(\alpha^{+}-\left.\right|_{-} \alpha^{-}-s^{-}\right)_{2}$ is 322 $\AA^{2} / \alpha$-helix, which is approximately half of the parallel $\alpha$-helices area. This value coincides with the configuration in which the four- $\alpha$-helix bundle lies parallel to the air-water interface but with two helices on top of the other two; it also indicates that the four- $\alpha$-helix-bundle structure remains integral and orients its helical axes parallel to the air-water interface.

Trace $\mathrm{f}$ in Figure 4B shows the isotherm of heme $2_{2}\left(\alpha^{+}-\mathrm{I}_{-} \alpha^{-}-\right.$ $\mathrm{s}-)_{2}$, and Figure 4D reveals that its $n$ value is 4.19 , which indicates as expected that the four $\alpha$-helices are associated in the independent particles on the air-water interface. In this case the $A_{0}$ value for heme $2_{2}\left(\alpha^{+}-\mid-\alpha^{-} \text {-s- }\right)_{2}$ is $194 \AA^{2} / \alpha$-helix and hence quite different from all other maquettes. This $A_{0}$ value is close to the expected cross section area of an $\alpha$-helix when it stands perpendicular to the air-water interface. This analysis indicates that the four- $\alpha$-helix-bundle structure of heme $2_{2}\left(\alpha^{+}-\mid-\alpha^{-}-\mathrm{s}^{-}\right)_{2}$ remains integral and orients perpendicular to the air-water interface.

Traces $g$ and $\mathrm{h}$ in Figure 4C show the isotherms of (palm ${ }_{2}^{-}$ $\alpha$-ss- $\alpha)_{2}$ and heme ${ }_{2}$ (palm $2-\alpha$-ss- $\left.\alpha\right)_{2}$ characterized in our previous paper. ${ }^{12}$ The low surface pressure region of the isotherms of $\left(\text { palm }_{2}-\alpha-\text { ss- } \alpha\right)_{2}$ and heme $2-\left(\text { palm }_{2}-\alpha-\text { ss- } \alpha\right)_{2}$ is very similar to that of $(\alpha-\text { ss- } \alpha)_{2}$ and heme $2-(\alpha-s s-\alpha)_{2}$. Figure 4D reveals that the $n$ value is 1.81 for (palm $2-\alpha-s s-\alpha)_{2}$ and 1.83 for heme $2^{-}$ (palm $2^{-} \alpha$-ss- $\left.\alpha\right)_{2}$, and the $A_{0}$ value is $590 \AA^{2} / \alpha$-helix for $\left(\right.$ palm $_{2-}$ $\alpha$-ss- $\alpha)_{2}$ and $598 \AA^{2} / \alpha$-helix for heme $2-\left(\text { palm }_{2}-\alpha \text {-ss- } \alpha\right)_{2}$. These indicate that at low surface pressure the number of helices associated as independent particles and helices orientation of $\left(\text { palm }_{2}-\alpha-s s-\alpha\right)_{2}$ and heme ${ }_{2}-\left(\text { palm }_{2}-\alpha-s s-\alpha\right)_{2}$ are similar to those of $(\alpha-\text { ss- } \alpha)_{2}$ and heme $2-(\alpha-\text { ss- } \alpha)_{2}$.

Remarkably, upon compression to high surface pressure, the isotherms of $\left(\text { palm }_{2}-\alpha-\text { ss- } \alpha\right)_{2}$ and heme ${ }_{2}-\left(\text { palm }_{2}-\alpha-s s-\alpha\right)_{2}$ display another phase transition that differs greatly from the isotherms of $(\alpha-\text { ss- } \alpha)_{2}$ and heme ${ }_{2}-(\alpha-\text { ss- } \alpha)_{2}$. This phase transition was suggested ${ }^{12}$ to correspond to the change of $\alpha$-helices orientation from parallel to perpendicular to the air-water interface (note that this high surface pressure region does not lend itself to analysis by eq 1). The surface area per $\alpha$-helix at this second phase transition is similar to the surface area per $\alpha$-helix of heme $_{2}-\left(\alpha^{+}-\mid-\alpha^{-}-s-\right)_{2}$ at the first phase transition (trace $f$ in Figure $4 \mathrm{~B})$. This match adds weight to the conclusion that both maquettes orient with $\alpha$-helix axes perpendicular to the airwater interface. The difference is that high surface pressures are required to lift $\left(\mathrm{palm}_{2}-\alpha-\mathrm{ss}-\alpha\right)_{2}$ and heme $2_{2}\left(\mathrm{palm}_{2}-\alpha-\mathrm{ss}-\alpha\right)_{2}$ out of the dissociated subunit stage with horizontal $\alpha$-helices into the vertical orientation, while heme $2_{2}\left(\alpha^{+}-\mid-\alpha^{-}-s^{-}\right)_{2}$ adopts the vertical orientation spontaneously.

3. Characterization of the Heme Protein Maquettes on LB Films. The heme protein maquettes heme $2^{-}(\alpha-\text { ss- } \alpha)_{2}$, heme $2^{-}$ $(\alpha-\mid-\alpha-s-)_{2}$, and heme ${ }_{2}-\left(\alpha^{+}-\mid-\alpha^{-}-s_{-}\right)_{2}$ were transferred to quartz substrates and studied by $\mathrm{UV}_{-}-\mathrm{vis}$ and $\mathrm{CD}$ spectra. UV-vis spectra revealed the heme ligation status, and CD spectra revealed the $\alpha$-helical secondary structure composition. CD 


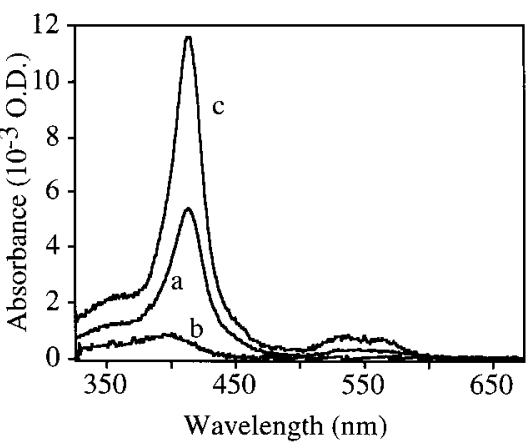

Figure 5. UV-vis absorption spectra of the heme protein maquette LB films. Spectra of LB films derived from heme $2^{-}(\alpha-s s-\alpha)_{2}(a)$, heme $_{2}-$ $(\alpha-l-\alpha-s-)_{2}(b)$, and heme ${ }_{2}-\left(\alpha^{+}-\mid-\alpha^{-}-s-\right)_{2}(c)$ are compared. Spectra were measured with two slides coated with a monolayer LB film on both sides, arranged perpendicularly to the measuring light beam.

spectra also indicated the $\alpha$-helical orientations, while UVvis linear dichroism revealed the orientation of the heme plane relative to the substrate plane.

Deposition Ratio. In the LB method, the relatively hydrophobic air side of the surface film, should adhere to a hydrophobic alkylated substrate upon immersion. However, the air-facing surface is not necessarily strongly hydrophobic, just less hydrophobic than the water-facing film surface; thus, deposition may not be perfect. The deposition ratio of heme $2_{2}$ $(\alpha \text {-ss- } \alpha)_{2}$ and heme ${ }_{2}-(\alpha-\mid-\alpha-s-)_{2}$ on transfer from the LB trough to the quartz slides was found to be close to unity, while the deposition ratio of heme $2^{-}\left(\alpha^{+}-\mid-\alpha^{-}-s_{-}\right)_{2}$ was not found to be so high; values in the range $0.6-0.8$ were typical.

Ultraviolet-Visible Spectra. The UV-vis spectra of heme $2_{2}$ $(\alpha-s s-\alpha)_{2}$, heme ${ }_{2}-(\alpha-\mid-\alpha-s-)_{2}$, and heme $2_{2}\left(\alpha^{+}-\mid-\alpha^{-}-s^{-}\right)_{2}$ in solution all display the same absorption properties characteristic of intact bis-histidine heme ligation in both the ferric and ferrous forms. The spectra of heme $2_{2}(\alpha-\text { ss- } \alpha)_{2}$ and heme $2_{2}\left(\alpha^{+}-\mid-\alpha^{-}-s_{-}\right)_{2}$ as LB films on quartz also display the characteristic UV-vis absorption properties of bis-histidine hemes (Figure 5), and they are identical to the spectra of the maquettes in solution (Figure 3B). Hence, it is evident that the bis-histidine heme ligation is conserved on these LB films. In contrast, the spectrum of LB film of heme $e_{2}(\alpha-\mid-\alpha-s-)_{2}$ differs from the others and shows absorption properties of unligated hemes (Figure 5), indicating loss of heme ligation on the film. These films were not analyzed further, and possible reasons for loss of heme will be discussed later.

The density of the bis-histidine ligated hemes of the heme $2^{-}$ $(\alpha \text {-ss- } \alpha)_{2}$ and heme $2^{-}\left(\alpha^{+}-\mid-\alpha^{-}-s_{-}\right)_{2}$ in the LB films was estimated from the absorbance of the Soret band maximum between 411 and $412 \mathrm{~nm}$, assuming the solution extinction coefficient $(\epsilon=$ $120000 \mathrm{M}^{-1} \mathrm{~cm}^{-1}$ ) applies and taking into account the deposition ratio. The heme density in the heme $-(\alpha-s s-\alpha)_{2}$ LB films (deposition ratio unity) was estimated to be $1230 \AA^{2} /$ bishistidine ligated heme. This is equivalent to $615 \AA^{2} / \alpha$-helix and directly consistent with the $602 \AA^{2} / \alpha$-helix value found by the isotherm on the air-water interface. The heme density in the heme $_{2}-\left(\alpha^{+}-\mid-\alpha^{-}-\mathrm{s}-\right)_{2}$ LB films was estimated to be $570 \AA^{2} / \mathrm{bis}-$ histidine ligated heme, which after correction for the deposition ratio (here measured to be 0.7 ; see earlier) is equivalent to 200 $\AA^{2} / \alpha$-helix, again consistent with the $194 \AA^{2} / \alpha$-helix value determined by the isotherm.

$C D$ Spectra. Figure 6 compares the CD spectra of heme $2^{-}$ $(\alpha \text {-ss- } \alpha)_{2},\left(\alpha^{+}-\mid-\alpha^{-}-s_{-}\right)_{2}$, and heme ${ }_{2}\left(\alpha^{+}-\mid-\alpha^{-}-s^{-}\right)_{2}$ LB films and their corresponding solution spectra. The CD spectra of the LB films conserve the shape that is characteristic of a predominately

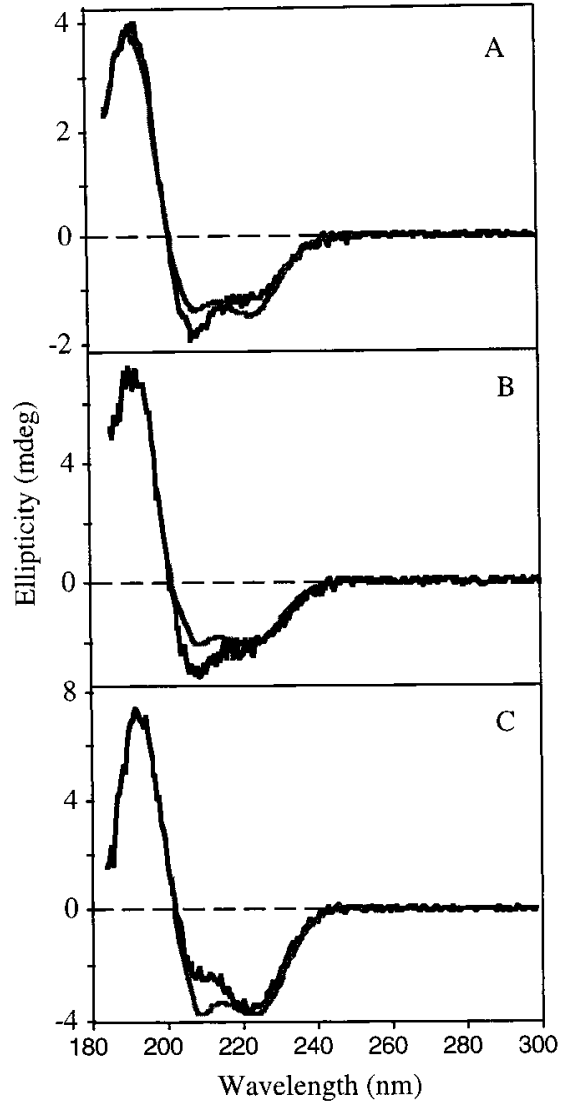

Figure 6. $\mathrm{CD}$ spectra of the maquette $\mathrm{LB}$ films and comparison with respective solution CD spectra: (A) heme $2_{2}(\alpha-s s-\alpha)_{2}$ LB film spectrum (dark line) and solution spectrum (gray line); (B) $\left(\alpha^{+}-\mid-\alpha^{-}-s^{-}\right)_{2}$ LB film spectrum (dark line) and solution spectrum (gray line); (C) heme $2_{2}\left(\alpha^{+}-\right.$ I- $\alpha^{-}$-s- $)_{2}$ LB film spectrum (dark line) and solution spectrum (gray line). LB film spectra were measured with four slides coated with monolayer LB film on both sides, arranged perpendicularly to the measuring light beam. The scales of the solution spectra were adjusted to compare with LB film spectra.

$\alpha$-helical structure. We note, however, that the ratio of two minima at 208 and $222 \mathrm{~nm}$ of the LB film spectra differs from that of the solution spectra. The CD band at $208 \mathrm{~nm}$ in the heme $_{2}-(\alpha-\text { ss- } \alpha)_{2}$ and $\left(\alpha^{+}-\mid-\alpha^{-}-\text {s- }\right)_{2}$ LB films is considerably more negative in comparison to their solution spectra, while the $208 \mathrm{~nm}$ band in the heme $2^{-}\left(\alpha^{+}-\mid-\alpha^{-}-\mathrm{s}^{-}\right)_{2} \mathrm{LB}$ film is considerably less negative in comparison to its solution spectrum

The Moffit exciton theory ${ }^{30,31}$ predicts that the band at 208 $\mathrm{nm}$ is polarized parallel to $\alpha$-helices. This prediction was proved by several experiments, including the work of Olah and Huang et al. ${ }^{32,33}$ and de Jongh et al. ${ }^{34,35}$ Since the incident light is normal to the substrate, a more negative $208 \mathrm{~nm}$ band corresponds to a predominantly parallel orientation of the helices with respect to the substrate surface and a less negative 208 $\mathrm{nm}$ band corresponds to a predominantly perpendicular orientation of the helices. Thus, the helices in heme $2-(\alpha-s s-\alpha)_{2}$ and $\left(\alpha^{+}-\mid-\alpha^{-}-\mathrm{s}-\right)_{2}$ LB films are predominantly parallel to the solid substrate while the helices in the heme $2_{2}\left(\alpha^{+}-\left.\right|_{-} \alpha^{-}-s_{-}\right)_{2}$ LB film are predominantly perpendicular to the substrate, results entirely consistent with their orientations identified by LB isotherms on the air-water interface.

Ultraviolet-Visible Linear Dichroism. The dichroic ratio of absorption of UV-vis light linearly polarized perpendicular versus parallel to the plane of incidence reveals the average tilt angle of the heme plane with respect to the substrate. It is generally assumed that the electronic transitions responsible for 


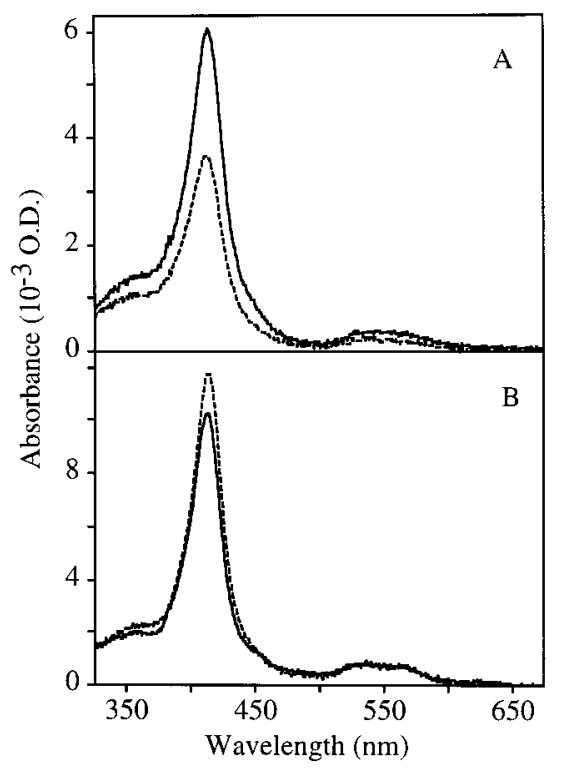

Figure 7. UV-vis linear dichroism of maquette LB films. Part A shows the absorption spectra of a monolayer heme $e_{2}(\alpha-s s-\alpha)_{2}$ LB film with incident measuring light polarized parallel (dashed line) and perpendicular (solid line) to the plane of incidence. The incident angle is $60^{\circ}$. Part $\mathrm{B}$ shows the absorption spectra of a monolayer heme $2^{-}$ $\left(\alpha^{+}-\mid-\alpha^{-}-s-\right)_{2}$ LB film with parallel (dashed line) and perpendicular (solid line) polarized incident measuring light. The incident angle is $60^{\circ}$.

the Soret and $\alpha$-bands in hemes are isotropically polarized in the plane of the heme. ${ }^{36}$ A relationship has been described ${ }^{37}$ to estimate the average orientation of the heme group in heme proteins.

$$
D=\cos ^{2} \theta+\frac{2 \sin ^{2} \psi}{1+\cos ^{2} \psi} \sin ^{2} \theta
$$

where $\theta$ is the incident angle of the incoming light on the LB film and $\psi$ is the angle between the normal of the heme plane and the normal of the substrate plane.

Figure 7 shows the UV-vis absorption spectra of a monolayer LB film of heme $2^{-}(\alpha-\text { ss- } \alpha)_{2}$ and heme $2^{-}\left(\alpha^{+}-\mid-\alpha^{-}-s-\right)_{2}$ with parallel and perpendicular polarized incident light and with an incident angle of $60^{\circ}$. The dichroic ratio of the heme ${ }_{2}-(\alpha-s s-$ $\alpha)_{2}$ LB film is 0.60 , corresponding to an average $38^{\circ}$ tilt angle of the heme plane relative the substrate plane. In contrast, the dichroic ratio of the heme ${ }_{2}-\left(\alpha^{+}-\mid-\alpha^{-}-\mathrm{s}-\right)_{2} \mathrm{LB}$ film is 1.14 , corresponding to an average $60^{\circ}$ tilt angle of the heme plane relative the substrate plane.

As shown in our previous paper, ${ }^{12}$ if we assume that the heme planes are parallel to the $\alpha$-helix axis, an average $38^{\circ}$ tilt angle of the heme plane implies that the average helix tilt angle is some value between $0^{\circ}$ and $38^{\circ}$, and likewise, an average $60^{\circ}$ tilt angle of the heme plane implies that the average helix tilt angle is between $0^{\circ}$ and $60^{\circ}$. Thus, we cannot infer the helix tilt angle from these heme plane tilt angles. Nevertheless, these results further support the $C D$ results that indicated that the helices in heme $2_{2}(\alpha-\text { ss- } \alpha)_{2}$ LB films are predominantly parallel to the solid substrate and those in heme ${ }_{2}\left(\alpha^{+}-\mid-\alpha^{-}-{ }^{-}\right)_{2}$ LB films are predominantly perpendicular to the substrate.

\section{Discussion}

Molecules at air-water interfaces are exposed to very strong surface tension forces quite different from the forces in bulk aqueous phase. These forces can have a profound effect on the

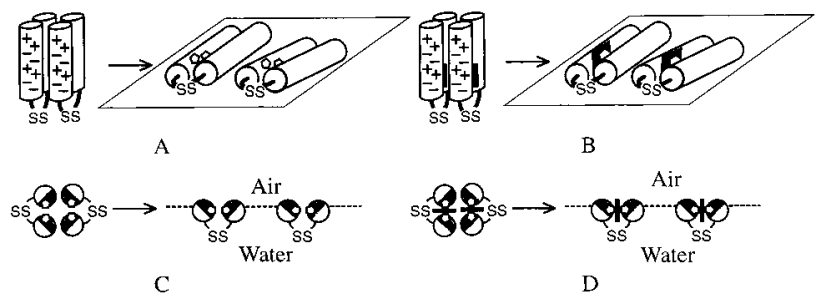

Figure 8. Illustrations of $(\alpha-s s-\alpha)_{2}$ and heme ${ }_{2}-(\alpha-s s-\alpha)_{2}$ maquettes transferred from aqueous solution to the air-water interface. The rhombuses in this figure and the following figures indicate the horizontal plane and do not represent the exact position of the air-water interface. Parts A $\left[(\alpha-s s-\alpha)_{2}\right]$ and B [heme $\left.2-(\alpha-s s-\alpha)_{2}\right]$ both show that the four$\alpha$-helix bundles dissociate into the $\alpha$-ss- $\alpha$ subunits with horizontally oriented helices when transferred to the air-water interface. The histidine pairs remain in proximity for heme binding. Parts $\mathrm{C}[(\alpha-\mathrm{ss}-$ $\alpha)_{2}$ ] and D [heme $\left.-(\alpha-s s-\alpha)_{2}\right]$ provide a view along the helix axis, in which the shaded regions represent the hydrophobic parts of the helices.

molecular structure of four- $\alpha$-helix bundles originally designed for stability in the bulk aqueous phase. While the hydrophobic force will drive bundle assembly in solution by minimizing the hydrophobic surface area exposed to solvent, at the air-water interface this force will be asymmetric and bundle formation is not ensured. Careful redesign can create molecules with both desirable solubility in the bulk aqueous phase and controlled self-assembly at the interface.

In the case of the original $(\alpha-\text { ss- } \alpha)_{2}$ maquettes (with or without hemes), the hydrophobic forces and spiral distribution of the charged residues driving bundle association in solution $\left(K_{\mathrm{d}} \approx 10^{-12} \mathrm{M}\right)$ does not ensure bundle assembly at the airwater interface. The hydrophobic air phase appears to successfully compete with the hydrophobic interactions of the four- $\alpha$ helix-bundle core to simply open up the hydrophobic interface between two $\alpha$-ss- $\alpha$ units and expose the hydrophobic face of each of the $\alpha$-ss- $\alpha$ to the air, as shown in Figure 8 (the shaded regions in parts $\mathrm{C}$ and $\mathrm{D}$ of Figure 8 represent the hydrophobic parts of the helices). However, because added heme binds internally in the $\alpha$-ss- $\alpha$ unit, the bis-histidine ligations of the hemes can be maintained on the air-water interface, as shown in parts B and D of Figure 8 .

In the case of $(\alpha-\mid-\alpha-s-)_{2}$ maquettes (with or without hemes), four- $\alpha$-helix-bundle formation is also overwhelmed on the airwater interface. However, the topological constraints of bundle association-dissociation in the covalently linked four- $\alpha$-helix bundle are much different than in the noncovalently linked $(\alpha$ ss- $\alpha)_{2}$. While the $(\alpha-s s-\alpha)_{2}$ four- $\alpha$-helix bundle can simply dissociate at the interface into $\alpha$-ss- $\alpha$ units that maintain histidines in proximity for heme binding (Figure 8), the covalently linked bundle can partially dissociate in several ways, only some of which maintain histidines in proximity for heme binding (Figure 9). The configuration shown in parts $\mathrm{A}$ and $\mathrm{B}$ of Figure 9 in principle can maintain binding of two hemes, and the configuration shown in parts $C$ and D of Figure 9 in principle can maintain binding of one heme between helices 2 and 3. It is clear that no significant binding of heme occurs (Figure 5). We suggest that interference from helices 1 and 4 force the histidine pair of helices 2 and 3 to point away from each other, causing the loss of the heme, as shown in parts $\mathrm{E}$ and $\mathrm{F}$ of Figure 9. An additional and possibly significant contributor to heme loss in a surface reorganization process is that a transient loss may be irreversible.

The dissociation of water-soluble bundles at the air-water interface can be conquered with several strategies. While the addition of hydrophobic tails to $(\alpha-s s-\alpha)_{2}$ maquettes, such as (palm $2-\alpha$-ss- $\alpha)_{2}$ maquettes (with or without hemes), did not 

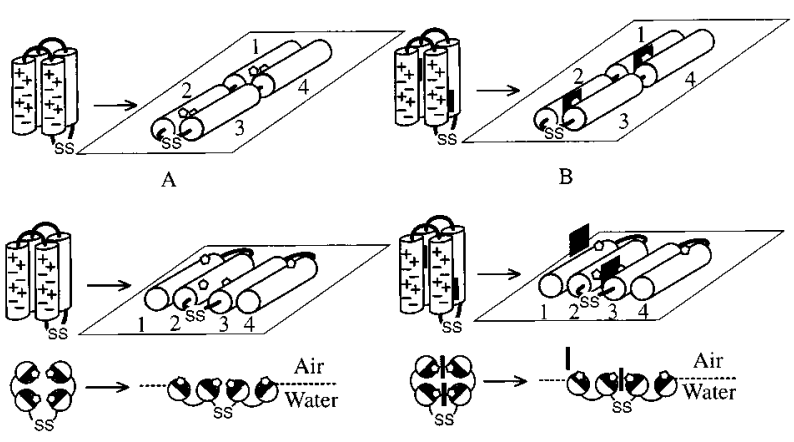

$\mathrm{D}$

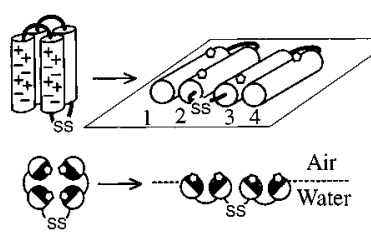

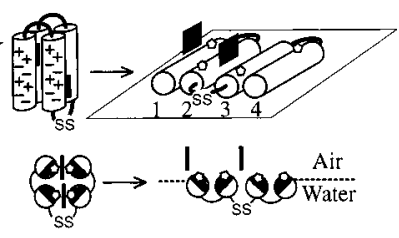

$\mathrm{F}$

Figure 9. Illustrations of $(\alpha-\mid-\alpha-s-)_{2}$ and heme $e_{2}(\alpha-\mid-\alpha-s-)_{2}$ maquettes transferred from aqueous solution to the air-water interface. Parts $\mathrm{A}$ $\left[(\alpha-\mid-\alpha-s-)_{2}\right]$ and $B$ [heme $\left.2-(\alpha-\mid-\alpha-s-)_{2}\right]$ show a dissociation topology of the four- $\alpha$-helix bundles at the air-water interface that maintains proximity between helices 1 and 4 and helices 2 and 3 and hence could permit full bis-histidine ligation of hemes. Parts $C\left[(\alpha-\mid-\alpha-s-)_{2}\right]$ and D $\left[\right.$ heme $\left.2-(\alpha-\mid-\alpha-s-)_{2}\right]$ show another dissociation topology of the four- $\alpha-$ helix bundles at the air-water interface, where the histidine pair provided by helices 1 and 4 is no longer in proximity while the pair provided by helices 2 and 3 is still in proximity for heme binding. Parts E $\left[(\alpha-\mid-\alpha-s-)_{2}\right]$ and $F\left[\right.$ heme $\left.2-(\alpha-\mid-\alpha-s-)_{2}\right]$ show that the histidine pair of helices 2 and 3 points away from each other to improve the packing between helices 1 and 2 and helices 3 and 4 . Bis-histidine ligation of hemes is not possible in this topology. In Parts $\mathrm{C}-\mathrm{F}$, views along the helix axis are also provided in which the shaded regions represent the hydrophobic parts of the helices.
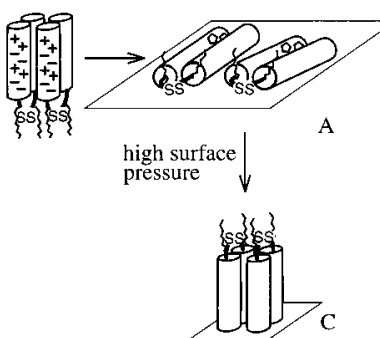
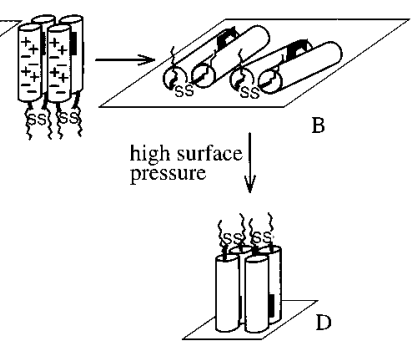

Figure 10. Illustrations of (palm $2-\alpha-s s-\alpha)_{2}$ and heme ${ }_{2}-\left(\right.$ palm $_{2}-\alpha-$ ss$\alpha)_{2}$ maquettes transferred from aqueous solution to the air-water interface. Parts A $\left[\left(\text { palm }_{2}-\alpha-\text { ss- } \alpha\right)_{2}\right]$ and B [heme $\left.2-\left(\text { palm }_{2}-\alpha-s s-\alpha\right)_{2}\right]$ show that the four- $\alpha$-helix bundles dissociate into the $\alpha$-ss- $\alpha$ subunits with horizontally oriented helices when transferred to the air-water interface, similar to $(\alpha-\text { ss- } \alpha)_{2}$ and heme $2-(\alpha \text {-ss- } \alpha)_{2}$ maquettes. Parts $C$ $\left[\left(\text { palm }_{2}-\alpha-s s-\alpha\right)_{2}\right]$ and D [heme $\left.2-\left(\text { palm }_{2}-\alpha-s s-\alpha\right)_{2}\right]$ show that at high pressure the bundles are forced into the water phase and anchored at the interface by the hydrophobic tails sticking out in the air phase.

prevent the four- $\alpha$-helix bundles from being everted by the surface forces at low surface pressure, as shown in parts $A$ and B of Figure 10, at high surface pressure the four- $\alpha$-helix bundles may be reassembled. Apparently, at high pressure the bundles are forced into the water phase, like the tail-free $(\alpha \text {-ss- } \alpha)_{2}$, but anchored at the interface by the hydrophobic tails sticking out in the air phase, shown in parts C and D of Figure 10.

The $\left(\alpha^{+}-\mid-\alpha^{-}-\mathrm{s}^{-}\right)_{2}$ maquettes represent a design that can resist the everting forces at the air-water interface even at low surface pressure. In this design, like charges were segregated onto different helices of the four- $\alpha$-helix bundles to create strong attractive forces between adjacent helices. These external

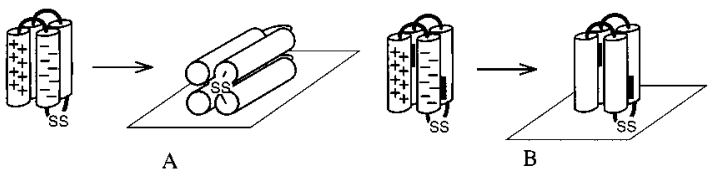

Figure 11. Illustrations of $\left(\alpha^{+}-\mid-\alpha^{-}-s^{-}\right)_{2}$ and heme ${ }_{2}\left(\alpha^{+}-\mid-\alpha^{-}-s^{-}\right)_{2}$ maquettes transferred from aqueous solution to the air-water interface. Part A shows that $\left(\alpha^{+}-\mid-\alpha^{-}-s-\right)_{2}$ remain as four- $\alpha$-helix bundles at the air-water interface, with the helix axis parallel to the interface. Part B shows that heme $2_{2}\left(\alpha^{+}-\mid-\alpha^{-}-s-\right)_{2}$ remain as four- $\alpha$-helix bundles at the air-water interface, with the helix axis perpendicular to the interface.

interhelix electrostatic attractions add to the hydrophobic forces driving bundle formation and create stable water-soluble maquette frames even at surfaces.

Yet another strategy involves the use of internal cofactors. The $\left(\alpha^{+}-\mid-\alpha^{-}-s^{-}\right)_{2}$ maquette with and without hemes were found to have different orientations on air-water interfaces, as shown in parts A and B of Figure 11. At the surface there will be a balance between two forces: electrostatic forces, which will tend to rotate helices to maximize the attractive interaction between complementary charged surfaces, and hydrophobic forces, which will tend to minimize the exposure of the hydrophobic residues of these helices to the aqueous subphase. Introducing the largely hydrophobic heme cofactor may significantly shift this balance to favor the geometry of Figure 11B, with helices oriented more perpendicular to the film surface. In another view of this balance, protein repacking upon heme binding may shift the distribution of partly exposed hydrophobic residues of the core to favor an orientation with one end of the bundle exposed to the air.

Segregation of like charges onto different helices is in a sense an extreme design because the attractive forces between the helices are so strong that under low ionic strength conditions they cause the bundles to aggregate. However, charge segregation is a common design in nature where relatively large patches of like charges are distributed on one side of a protein, such as cytochrome $c$ and plastocyanin. This kind of charge distribution usually promotes the binding of proteins to an oppositely charged surface. The modification of the charged residues distribution in de novo designed proteins offers an engineering technique to control the assembly of proteins not only at the air-water interface but on surfaces in general.

Acknowledgment. This research was supported by a grant from NIH GM41048 and in part by an MRSEC/IRG grant from NSF (DMR96-20668).

\section{References and Notes}

(1) DeGrado, W. F.; Wasserman, A. R.; Lear, J. D. Science 1989, 243 , $622-628$

(2) Bryson, J. W.; Betz, S. F.; Lu, H. S.; Suich, D. J.; Zhou, H. X.; O’Neil, K. T.; DeGrado, W. F. Science 1995, 270, 935-941.

(3) Tuchscherer, G.; Scheibler, L.; Dumy, P.; Mutter, M. Biopolymers 1998, 47, 63-73.

(4) Moser, C. C.; Keske, J. M.; Warncke, K.; Farid, R. S.; Dutton, P. L. Nature 1992, 355, 796-802.

(5) Bendall, D. S., Ed. Protein Electron Transfer; BIOS Scientific Publishers Ltd.: Oxford, 1996.

(6) Robertson, D. E.; Farid, R. S.; Moser, C. C.; Urbauer, J. L.; Mulholland, S. E.; Pidikiti, R.; Lear, J. D.; Wand, A. J.; DeGrado, W. F.; Dutton, P. L. Nature 1994, 368, 425-432.

(7) Choma, C. T.; Lear, J. D.; Nelson, M. J.; Dutton, P. L.; Robertson, D. E.; DeGrado, W. F. J. Am. Chem. Soc. 1994, 116, 856-865.

(8) Rabanal, F.; DeGrado, W. F.; Dutton, P. L. J. Am. Chem. Soc. 1995, $118,473-474$.

(9) Gibney, B. R.; Rabanal, F.; Reddy, K. S.; Dutton, P. L. Biochemistry 1998, 37, 4635-4643.

(10) Shifman, J. M.; Moser, C. C.; Kalsbeck, W. A.; Bocian, D. F.; Dutton, P. L. Biochemistry 1998, 37, 16815-16827. 
(11) Pilloud, D. L.; Rabanal, F.; Gibney, B. R.; Farid, R. S.; Moser, C. C.; Dutton, P. L. J. Phys. Chem. B 1998, 102, 1926-1937.

(12) Chen, X.; Moser, C. C.; Pilloud, D. L.; Dutton, P. L. J. Phys. Chem. B 1998, 102, 6425-6432.

(13) Song, S.; Clark, R. A.; Bowden, E. F.; Tarlov, M. J. J. Phys. Chem. 1993, 97, 6564-6572.

(14) Armstrong, F. A.; Heering, H. A.; Hirst, J. Chem. Soc. Rev. 1997, $26,169-179$.

(15) Pilloud, D. L.; Rabanal, F.; Moser, C. C.; Dutton, P. L. Biophys. J. 1998, 74 (2), A250.

(16) Nicolini, C. Biosens. Bioelectron. 1995, 10, 105-127.

(17) Jortner, J.; Ratner, M. A., Eds. Molecular Electronics; Blackwell Science: London, 1997.

(18) Marx, K. A.; Samuelson, L. A.; Kamath, M.; Lim, J. O.; Sengupta, S.; Kaplan, D.; Kumar, J.; Tripathy, S. K. Mol. Biomol. Electron. 1994, 240, 395-412.

(19) Mrksich, M.; Whitesides, G. M. Annu. Rev. Biophys. Biomol. Struct. 1996, 25, 55-78.

(20) Gibney, B. R.; Rabanal, F.; Skalicky, J. J.; Wand, A. J.; Dutton, P. L. J. Am. Chem. Soc. 1999, 121, 4952-4960.

(21) Grosset, A. M.; Rabanal, F.; Dutton, P. L. Manuscript in preparation. (22) Grosset, A. M.; Rabanal, F.; Farid, R. S.; Robertson, D. E.; Pilloud, D. L.; DeGrado, W. F.; Dutton, P. L. Peptides: Chemistry, Structure, and Biology; Kaumaya, P. T. P., Hodges, R. S., Eds.; Mayflower Scientific Ltd.: England, 1996; pp 573-574.

(23) Gibney, B. R.; Johansson, F. S.; Rabanal, F.; Skalicky, J. J.; Wand, A. J.; Dutton, P. L. Biochemistry 1997, 36, 2798-2806.
(24) Skalicky, J. J.; Gibney, B. R.; Rabanal, F.; Bieber-Urbauer, R. J.; Dutton, P. L.; Wand, A. J. J. Am. Chem. Soc. 1999, 121, 4941-4951.

(25) Gibney, B. R.; Huang, S. S.; Skalicky, J. J.; Fuentes, E. J.; Wand,

A. J.; Dutton, P. L. Manuscript in preparation.

(26) Popovic, Z. D.; Kovacs, G. J.; Vincett, P. S.; Alegria, G.; Dutton,

P. L. Chem. Phys. 1986, 110, 227-237.

(27) Popovic, Z. D.; Kovacs, G. J.; Vincett, P. S.; Alegria, G.; Dutton, P. L. Biochim. Biophys. Acta 1986, 851, 38-48.

(28) Trurnit, H. J. J. Colloid Interface Sci. 1960, 15, 1-13.

(29) Adamson, A. W.; Gast, A. P. Physical Chemistry of Surfaces, 6th ed.; John Wiley \& Sons: New York, 1997; Chapters 2 and 4.

(30) Moffitt, W. J. Chem. Phys. 1956, 25, 467.

(31) Woody, R. W. In The Peptides; Udenfriend, S., Meienhofler, J., Eds.; Academic: New York, 1985 and references therein.

(32) Olah, G. A.; Huang, H. W. J. Chem. Phys. 1988, 89, 2531-2538.

(33) Wu, Y.; Huang, H. W.; Olah, G. Biophys. J. 1990, 57, 797-806.

(34) de Jongh, H. H. J.; Goormaghtigh, E.; Killian, J. A. Biochemistry 1994, 33, 14521-14528.

(35) de Jongh, H. H. J.; Brasseur, R.; Killian, J. A. Biochemistry 1994, $33,14529-14535$.

(36) Adar, F. In The Porphyrins; Dolphin, D., Ed.; Academic Press: New York, 1978; Vol. III, pp 167-206.

(37) Blasie, J. K.; Erecinska, M.; Samuels, S.; Leigh, J. S. Biochim. Biophys. Acta 1978, 501, 33-52. 